\title{
VARIATION OF ELECTRIC PROPERTIES BETWEEN SURFACE PERMANENT MAGNET AND INTERIOR PERMANENT MAGNET MOTOR
}

\author{
BYUNG-CHUL WOO \\ Electric Motor Research Center, Korea Electrotechnology Research Institute, 28-1 Sungju-dong, Changwon, \\ KyungNam, 641-120, Korea \\ bcwoo@keri.re.kr \\ DO-KWAN HONG \\ Electric Motor Research Center, Korea Electrotechnology Research Institute, 28-1 Sungju-dong, Changwon, \\ KyungNam, 641-120, Korea \\ dkhong@keri.re.kr \\ JI-YOUNG LEE \\ Electric Motor Research Center, Korea Electrotechnology Research Institute, 28-1 Sungju-dong, Changwon, \\ KyungNam, 641-120, Korea \\ jylee@keri.re.kr
}

\begin{abstract}
The most distinctive advantage of transverse flux motor(TFM) is high torque density which has prompted many researches into studying various design variants. TFM is well suited for low speed direct drive applications due to its high torque density. This paper deals with simulation based comparisons between a surface permanent magnet transverse flux motor(SPM-TFM) and an interior permanent magnet transverse flux motor(IPM-TFM). A commercial finite element analysis(FEA) software Maxwell 3D is used for electromagnetic field computation to fully analyze complex geometry of the TFMs. General characteristics, such as cogging torque, rated torque and torque ripple characteristics of the two TFMs are analyzed and compared by extensive 3D FEA.

Keywords: Surface Permanent Magnet Motor; Interior Permanent Magnet Motor; Transverse Flux Motor; Cogging Torque.
\end{abstract}

\section{Transverse flux machine}

The study of transverse flux motor(TFM) started at T. U. Braunshweig in 1985. The TFMs are basically single-phase electric machine with ring winding per phase in stator, which is embraced by U-shaped cores. Rotor consists either of surface mounted PMs or interior PMs. TFM topology increases number of poles without reducing magneto-motive force(MMF) per pole, it is able to generate higher power densities than the conventional electric machines. During the following 10 years, a number of research projects with different magnetic machine in transverse flux mode, aiming at different application like 
electric car, wind power generation, direct driver for the several types of traction machine. Despite its distinctive advantages, such as high power density and specific torque density, the TFM is still considered as a newcomer in the class of special electric machines and no mass production was reported yet.

For an electric machine, to obtain tangential force component in a rotor, magnetic flux and applied current must flow perpendicular to each other and the magnetic flux must flow across air-gap perpendicularly to iron core surface. Therefore, the flux has to flow either parallel (longitudinally) or perpendicularly (transversely) to the moving direction of the rotor. For TFM topology the flux flows perpendicularly to the moving direction. Fig. 1 schematically illustrates flux flow of TFM and longitudinal flux machine(LFM). It can be seen in Fig. 1(a) that the flux(dotted line) flows perpendicular to applied current(arrow) as the mover moves along the same direction as the applied current.

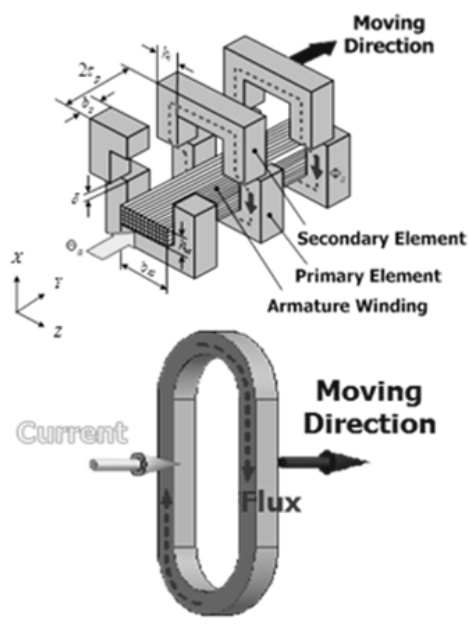

(a) Transverse flux machine
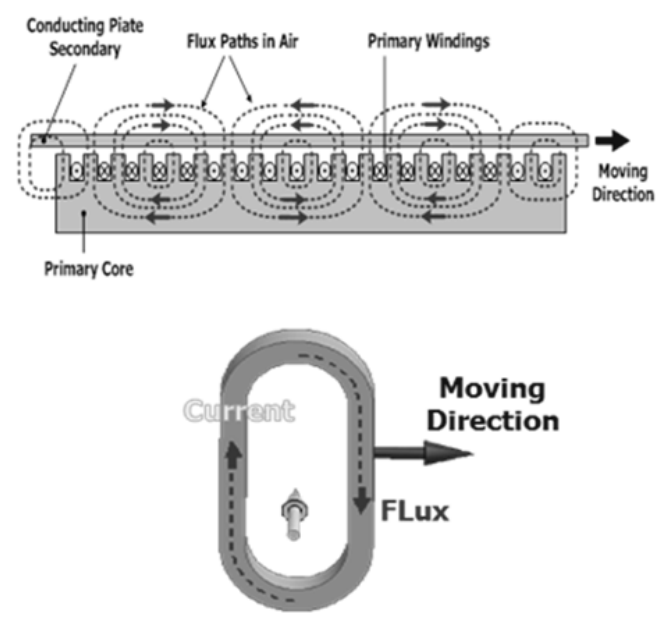

(b) Longitudinal flux machine

Fig. 1. The configuration of transverse flux machine and longitudinal flux machine.

\section{Properties of transverse flux motors with SPM and IPM}

This paper deals with two types of outer rotor TFM for direct driver applications. The one is a surface mounted permanent magnet transverse flux motor(SPM-TFM) and the other is an interior permanent magnet of transverse flux motor(IPM-TFM). The prototypes of the two TFMs are shown as Fig. 2 and the stator shape of each type is similar. Two different types of TFMs were developed for low speed high torque applications. General features of the two TFMs are given together with some simulation comparisons in this section. 


\subsection{SPM-TFM}

The surface mounted permanent magnet TFM(SPM-TFM) is shown in Fig. 2(a) which has PMs of alternating polarities on the inner surface of the rotor. Smooth torque profile can be achieved with SPM-TFM topology when cogging torque reduction design is adequately implemented.

\subsection{IPM-TFM}

The interior permanent magnet transverse flux motor is shown in Fig. 2(b) which adopts flux concentration structure in the rotor. With the flux concentration structure, airgap flux density of IPM-TFM increases and thus higher torque. On top of that, this structure helps to utilize additional reluctance torque which also leads to torque increase. Fig. 2 shows rotors of the SPM-TFM and the IPM-TFM which are compared in this paper.

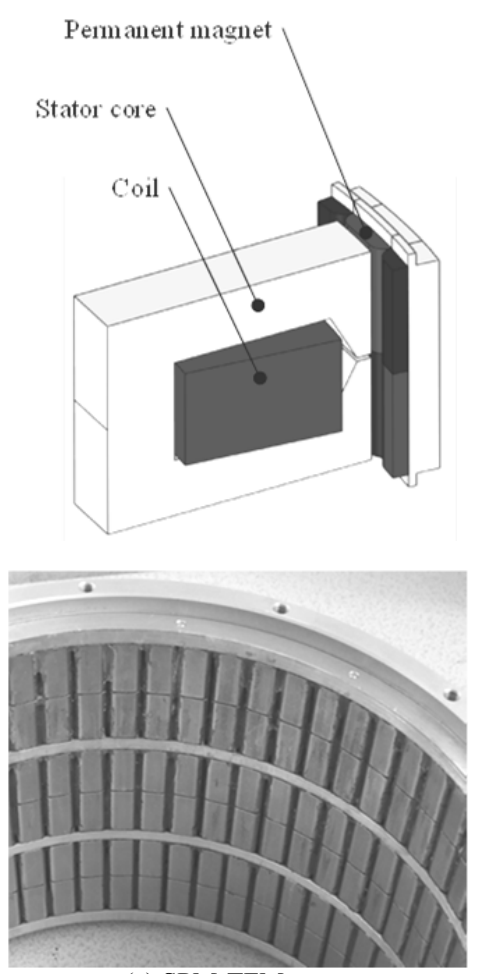

(a) SPM-TFM
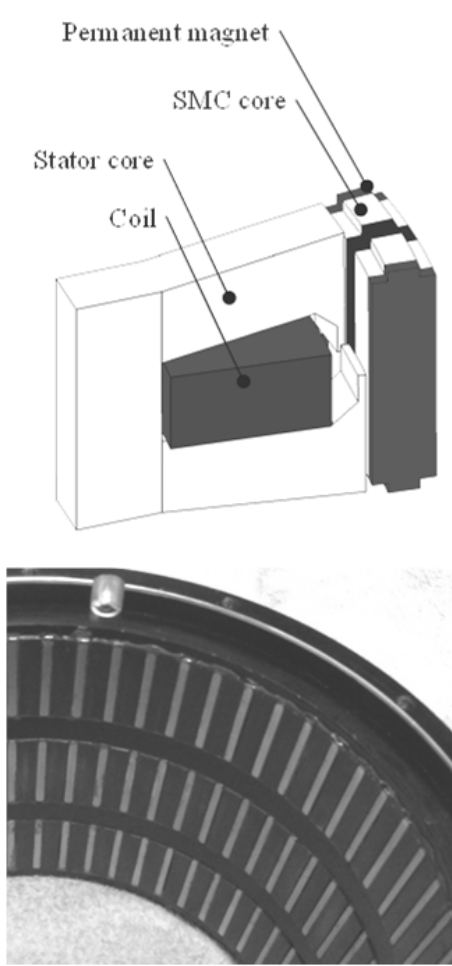

(b) IPM-TFM

Fig. 2. The rotor structure of SPM-TFM and IPM-TFM

\subsection{FEA results and magnetic flux density}

For 3D electromagnetic computation, a commercial FEA software Maxwell 3D is used to fully consider complex geometries of the TFMs. Considering geometric 
periodicity, one periodic model is more than enough for the field analysis. For the boundary conditions, the one periodic model of each TFM is enclosed by wedge-shape air space and master and slave boundary condition is applied on each side plane of the wedge. For the laminated stator of the TFMs, S23 is used and SMC(Somaloy 700)is used for the rotor flux concentration structure of IPM-TFM. Fig. 3 shows no load flux density distributions of periodic model of the TFMs. The average value of magnetic flux density is near of $1.5[\mathrm{~T}]$ but maximum value of magnetic flux density reaches up to $3[\mathrm{~T}]$.

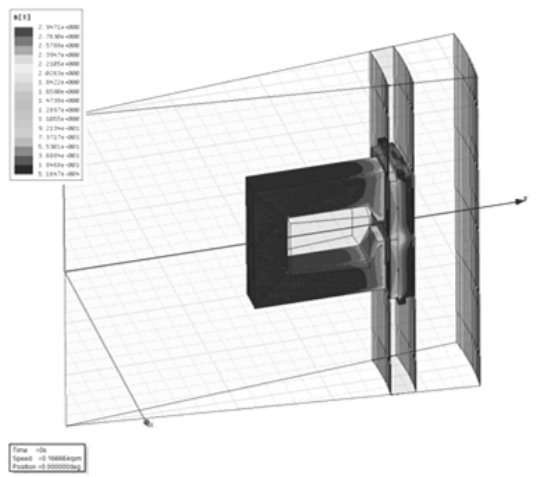

(a) SPM-TFM

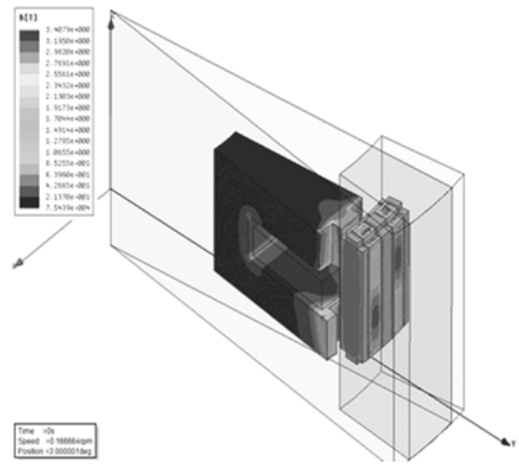

(b) IPM-TFM

Fig. 3. The magnetic flux density of the transverse flux rotary machine.

\subsubsection{Cogging torque}

Cogging torque waveforms of two types are compared in Fig. 4. Even though each phase module generates huge cogging torque, the resultant cogging torque by all 3-phase decreases dramatically since cogging torque generated by each phase module is quite sinusoidal. Consequently, peak cogging torque of the SPM-TFM reduces down to $0.7[\mathrm{Nm}]$ and that of IPM-TFM reduces down to $1.1[\mathrm{Nm}]$.

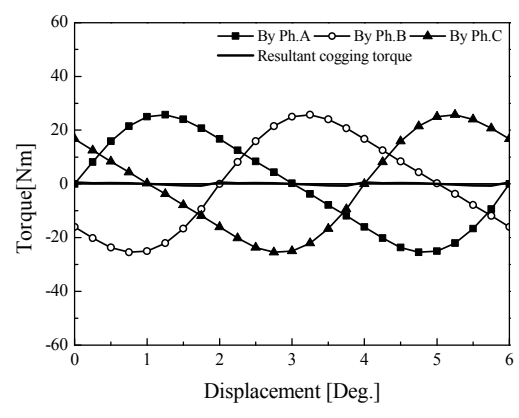

(a) SPM-TFM

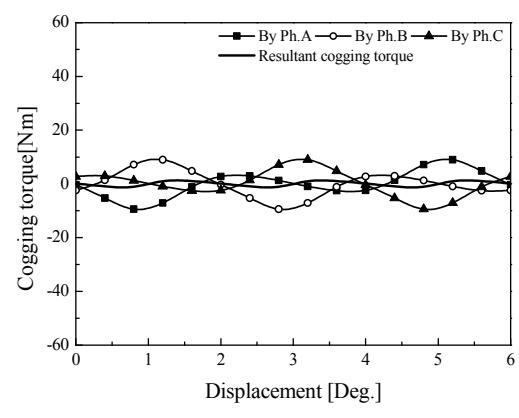

(b) IPM-TFM

Fig. 4. The cogging torque of the transverse flux rotary machine. 


\subsubsection{Rated torque}

Rated torque of the two models are computed and compared in Fig. 5 which shows SPM-TFM generates $75[\mathrm{Nm}]$ while IPM-TFM generates $115[\mathrm{Nm}]$. It is obvious that IPM-TFM generates higher torque than SPM-TFM due to flux concentration and additional reluctance torque. However, it can be stated that the SPM-TFM is advantageous over the other. In this particular comparison, SPM-TFM shows torque ripple of $\pm 2.6 \%$ while the other suffers torque ripple of $\pm 5.9 \%$.

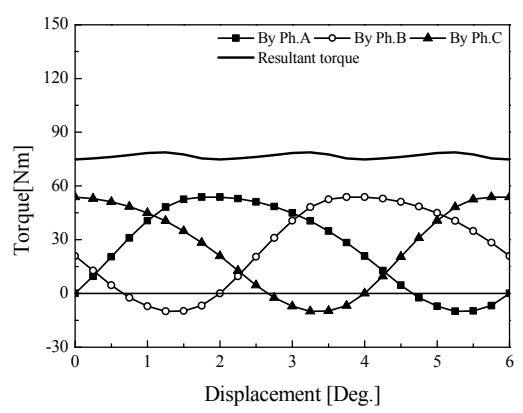

(a) SPM-TFM

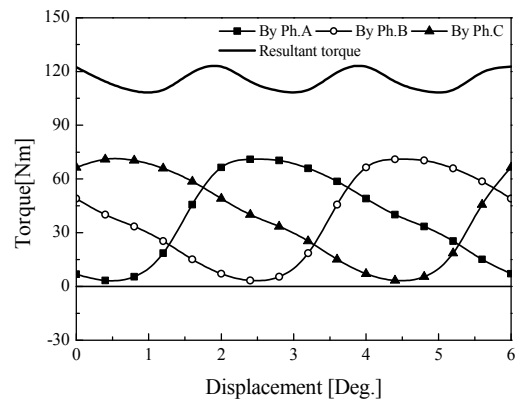

(b) IPM-TFM

Fig. 5. The rated torque of the transverse flux rotary machine.

\section{Test results}

Fig. 6(a) shows static torque measurement for IPM-TFM with respect to MMF and relative rotor position under DC current excitation. Fig. 6(b) shows dynamic torque measurement with respect to RPM and current when driven by inverter. However, experiments on SPM-TFM have yet to be followed to evaluate performance.

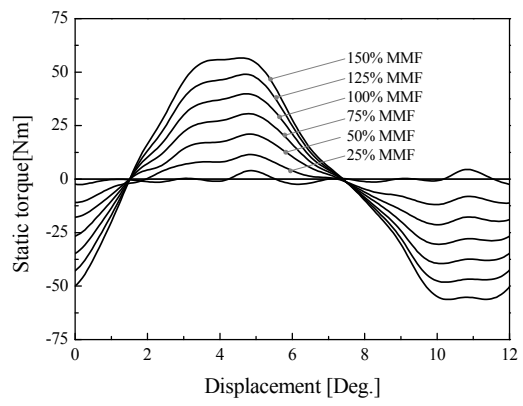

(a) Static torque

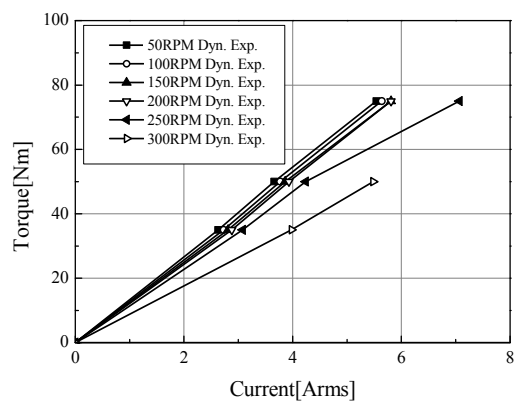

(b) Dynamic torque

Fig. 6. Test results of IPM-TFM.

\section{Conclusion}

Two different TFMs were introduced and analyzed and compared. Both of them are suitable for low speed high torque applications. Considering torque characteristics, SPM- 
TFM is suited for applications which require low torque ripple while IPM-TFM is suited for applications which require high specific torque.

\section{References}

1. W. M. Arshad and T. Backstrom, "Analytical design and analysis procedure for a transverse flux machine", IEEE. Int. Conf. on Electric Machines and Derivers, 2001, pp.115-121

2. H. Weh, "New permanent magnet excited synchronous machine with high efficiency at low speeds", Proc. Int. Conf. on Electric Machines, 1988, pp.35-40

3. D. H. Kang, "A study on the design of transverse flux linear motor with high power density", IEEE. Int. Sym. Industrial Electronics, 2001, pp.707-711

4. B.C. Woo et al., IEEE Transactions on Magnetics Vol.43, No.6, 2007, pp.2576-2578

5. D.K. Hong et al., Journal of Applied Physics Vol.103, No.7, 2008, 07F120

6. D.K. Hong et al., IEEE Transactions on Magnetics Vol.45, No.6, 2009, pp.1-4 